\title{
Multiple carrier frequency offset compensation for cooperative HAPS IoT systems
}

\author{
Yanyan Zhang* ${ }^{*}$, Baocong Wang, Xing Li, Na Liu, Wenjuan Ji and Xin Li
}

\author{
*Correspondence: zhangyanyan@ \\ cmdi.chinamobile.com \\ China Mobile Group Design \\ Institute Co., Ltd, Beijing, China
}

\begin{abstract}
Due to the deployment flexibility and broad coverage capabilities, High Altitude Platform Station is an efficiency solution of communication access for underserved areas like remote areas. However, for some special scenarios, multiple platforms may need to collaborate to overcome the effects of terrain. But the interferences introduced by multiple carriers and access links may hinder the process and restrict its practicability. In order to eliminate these interferences, a proposal on multiple carrier frequency offsets (MCFO) compensation algorithm was provided in this paper. Furthermore, this paper analyzed the lower bound of bit error ratio (BER) for cooperative HAPS systems. Simulation was also carried out, and the results validate the correctness of our theoretical analysis results.

Keywords: Multiple carrier frequency offsets, Internet of Things, High Altitude Platform Station, Inter carrier interference, Multiple access interference, Minimum Euclidean Distance Decision Criterion
\end{abstract}

\section{Springer Open}

\section{Introduction}

Due to the lack of telecommunications infrastructure and high density of trees and peaks, the signal coverage of rural and remote areas are restricted. However, these areas occupy an equally important position in the development of the country especially in Internet of Things (IoT) [1]. In many countries, rural and remote areas play an important role, such as protecting natural resources, forest fire prevention, in which the IoT network can play a crucial part. Therefore, the network should cover a wide range by employing huge number of base stations, and it will raise the network cost significantly.

In this case, special telecommunication technologies are required in rural and remote areas [2], especially because of their unique isolation environment, few population, large terrain, and inability to make productive investments [3]. Although various telecommunication methods have been proposed for rural areas where these services are lacking, efforts are needed to achieve Internet and cellular access [4].

(c) The Author(s). 2020 Open Access This article is licensed under a Creative Commons Attribution 4.0 International License, which permits use, sharing, adaptation, distribution and reproduction in any medium or format, as long as you give appropriate credit to the original author(s) and the source, provide a link to the Creative Commons licence, and indicate if changes were made. The images or other third party material in this article are included in the article's Creative Commons licence, unless indicated otherwise in a credit line to the material. If material is not included in the article's Creative Commons licence and your intended use is not permitted by statutory regulation or exceeds the permitted use, you will need to obtain permission directly from the copyright holder. To view a copy of this licence, visit http://creativecommons.org/licenses/by/4.0/. 
Common rural telecommunication [5] infrastructure solutions are VSAT (Very Small Aperture Terminal) [6, 7] and microwave radio. Common solutions such as VSAT [8] and microwave links [9] bring high investment and operating costs, such as on-site acquisition, on-site visits, and technology costs, and are faced with low user density and low affordability in the return. Many operators therefore invest less in their development.

High Altitude Platform Station (HAPS) $[10,11]$ is also known as stratosphere repeaters. Traditional ground systems have problems like small coverage and strict propagation environment. However, these problems can be eliminated by using HAPS systems [12, 13]. It is a new approach to telecommunication infrastructure solutions in rural and remote areas based on the stratospheric airborne platform. So HAPS is very suitable for Internet of Things applications in remote areas.

However, in some special scenarios, the coverage of HAPS may be affected due to the obstacle from topography such as mountains and forests [14] and the communication between the platform and the device. Coordination between multiple HAPS stations may be need in these scenarios. However, there are interference challenges between multiple HAPS stations in the cooperative HAPS systems due to inter-carrier interference (ICI) and multiple access interference (MAI).

Orthogonal frequency division multiplexing (OFDM) is a basic technology for IoT systems, such as NB-IoT. In OFDM-based IoT systems [15], the channels are divided into orthogonal sub channels, and the data is converted into several parallel data streams, which are modulated to the sub channels for transmission. Multiple carrier frequency offsets (MCFO) [16] may result in ICI [17] and MAI [18] and will hinder the synchronization between the device and HAPS.

In this paper, we propose a new frequency offset compensation algorithm for HAPS IoT systems employing space-frequency block code orthogonal frequency division multiplexing (SFBC-OFDM), which can provide nearly the ideal performance under certain conditions. Furthermore, the performance of the algorithm was analyzed, and simulation was also carried out to verify the result.

This paper is organized as description in the follows. The system model and the proposed MCFO compensation algorithm are depicted in Section II. Section III describes the theoretical analysis on BER (bit error ratio) performance and the simulation results, and finally the conclusion is depicted in section IV.

\section{Methods}

\subsection{System model}

The system model is defined in this sub-section. In this paper, we assumed that two HAPS stations collaborate to provide coverage for the same terminal through SFBCOFDM mode. In SFBC-OFDM mode, two HAPS stations can be divided into main station and assist station. To simplify the system model, the system model can be abstracted as a relay system, in which the main HAPS is the source node and the assist HAPS is the relay node, as shown in Fig. 1.

All stations and device work in half duplex mode. The cooperative HAPS systems

will listen and then transmit to the device. When listening, the source HAPS 

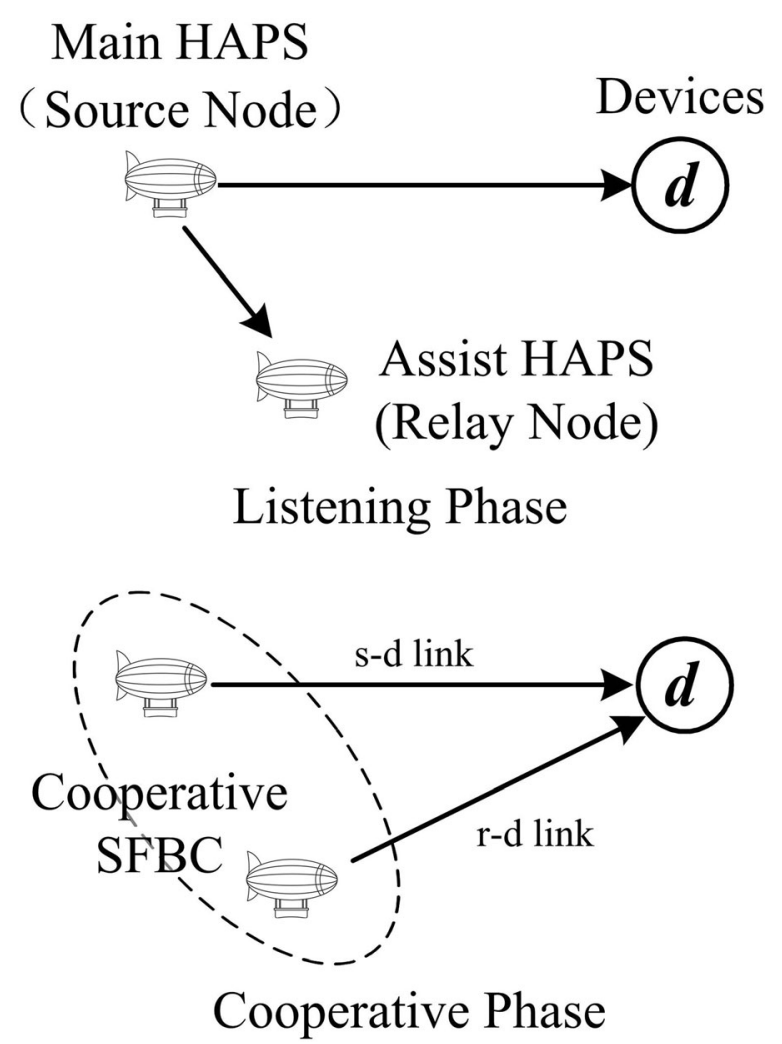

Fig. 1 System model. Figure 1 shows the communication links among main HAPS, assist HAPS, and devices in two phases

broadcasts the information to the assist HAPS and the device. And we assume that the transmitted data can be ideally detected by the assist HAPS. When transmitting, the two HAPS can transmit the data to the device cooperatively. In this paper, we assume that SFBC is employed. According to the SFBC scheme [19] that employs Altamonte strategy [20], the encoded vectors corresponding to the two nodes can be expressed as:

$$
\begin{aligned}
& \mathbf{X}_{s d}=\left[X_{0},-X_{1}^{*}, \cdots, X_{k},-X_{k+1}^{*}, \cdots, X_{N-2},-X_{N-1}^{*}\right]^{T} \\
& \mathbf{X}_{r d}=\left[X_{1}, X_{0}^{*}, \cdots, X_{k+1}, X_{k}^{*}, \cdots, X_{N-1}, X_{N-2}^{*}\right]^{T}
\end{aligned}
$$

Then, the coded data are both OFDM modulated as $\mathbf{x}_{\alpha}=\mathbf{F}_{N}^{-1} \mathbf{X}_{\alpha}(\alpha \in\{s d, r d\})$, where $\mathbf{F}_{N}^{-1}$ is the inverse Fourier matrix. Finally, a cyclic prefix (CP) is added to the top of $\mathbf{x}_{\alpha}$, which is utilized to eliminate the inter-symbol interference (ISI). And $N_{g}$ is the length of the CP.

The discrete time model of the received signal after removing the $\mathrm{CP}$ is as described in formula (2)

$$
\mathbf{y}_{d}=\sum_{\alpha=s d, r d} \boldsymbol{\Xi}_{\alpha} \cdot \mathbf{x}_{\alpha} \cdot \mathbf{h}_{\alpha}+\mathbf{w}_{d}
$$

where $L$ is the length of the frequency selective channel 


$$
\begin{aligned}
& \mathbf{y}_{d}=\left[y_{d}(0), y_{d}(1), \cdots, y_{d}(N-1)\right]^{T}, \\
& \mathbf{h}_{\alpha}=\operatorname{diag}\left\{\mathbf{h}_{\alpha}(0), \mathbf{h}_{\alpha}(1), \cdots, \mathbf{h}_{\alpha}(N-1)\right\}, \\
& \mathbf{h}_{\alpha}(n)=\left[h_{\alpha}(n, 0), \cdots, h_{\alpha}(n, L-1)\right], \\
& \boldsymbol{\Xi}_{\alpha}=\operatorname{diag}\left\{\boldsymbol{\Xi}_{\alpha}(0), \boldsymbol{\Xi}_{\alpha}(1), \cdots, \boldsymbol{\Xi}_{\alpha}(N-1)\right\}, \\
& \boldsymbol{\Xi}_{\alpha}(n)=\operatorname{diag}\{\underbrace{e^{j \frac{2 \pi \cdot \varepsilon_{\alpha} \cdot n}{N}}, \cdots, e^{j \frac{2 \pi \cdot \varepsilon_{\alpha} \cdot n}{N}}}_{L}\} \\
& \mathbf{w}_{\alpha}=\left[w_{\alpha}(0), w_{\alpha}(1), \cdots, w_{\alpha}(N-1)\right]^{T} .
\end{aligned}
$$

In consequence, the frequency domain received signals can be expressed as

$$
\begin{aligned}
& \mathbf{Y}_{d} \\
& =\left[\begin{array}{c}
\boldsymbol{\Lambda}_{s d} \\
\boldsymbol{\Lambda}_{r d}
\end{array}\right]^{T} \cdot\left[\begin{array}{cc}
\operatorname{diag}\left\{\mathbf{H}_{s d}\right\} & 0 \\
0 & \operatorname{diag}\left\{\mathbf{H}_{r d}\right\}
\end{array}\right] \cdot\left[\begin{array}{c}
\mathbf{X}_{s d} \\
\mathbf{X}_{r d}
\end{array}\right]+\mathbf{W}_{d} \\
& =\left(\left[\begin{array}{c}
\boldsymbol{\Lambda}_{\varepsilon_{s d}, 0} \\
\boldsymbol{\Lambda}_{\varepsilon_{r d}, 0}
\end{array}\right]+\left[\begin{array}{c}
\boldsymbol{\Pi}_{s d} \\
\boldsymbol{\Pi}_{r d}
\end{array}\right]^{T}\right) \cdot\left[\begin{array}{cc}
\operatorname{diag}\left\{\mathbf{H}_{s d}\right\} & 0 \\
0 & \operatorname{diag}\left\{\mathbf{H}_{r d}\right\}
\end{array}\right] \cdot\left[\begin{array}{c}
\mathbf{X}_{s d} \\
\mathbf{X}_{r d}
\end{array}\right]+\mathbf{W}_{d}
\end{aligned}
$$

where $\mathbf{H}_{\alpha}(\alpha \in\{s d, r d\})$ is the diagonal channel transform function (CTF) matrix given by $\mathbf{H}_{\alpha}=\operatorname{diag}\left\{H_{\alpha}(0), H_{\alpha}(1), \cdots, H_{\alpha}(N-1)\right\}$, which is assumed to be unchanged during at least 2 sub-carriers. It is assumed that the CTF on every sub-carriers are independent, and they are Rayleigh random variables. $\boldsymbol{\Lambda}_{\alpha}$ denotes the ICI coefficient matrix caused by $\mathrm{MCFO}$, in which $\Lambda_{\alpha}[k, l]$ is defined as

$$
\begin{aligned}
& \Lambda_{\varepsilon_{\alpha}}[k, l]=\frac{1}{N} \cdot \sum_{n=0}^{N-1} e^{\frac{j 2 \pi n\left(\varepsilon_{\alpha}-k+l\right)}{N}} \\
& =\frac{\sin \left(\pi\left(l-k+\varepsilon_{\alpha}\right)\right)}{N \cdot \sin \left(\pi\left(l-k+\varepsilon_{\alpha}\right) / N\right)} \cdot \exp \left(j \pi\left(1-\frac{1}{N}\right)\left(l-k+\varepsilon_{\alpha}\right)\right)
\end{aligned}
$$

In which, $\varepsilon_{\alpha}$ is the normalized frequency offset. And it is obvious that when $k=l, \Lambda_{\varepsilon_{\alpha}}[k, l]$ is a constant value for fixed $\varepsilon_{\alpha}$, which is denoted as $\Lambda_{\varepsilon_{\alpha}, 0}$ and $\Lambda_{\varepsilon_{\alpha}, 0}=\operatorname{diag}\{\underbrace{\Lambda_{\varepsilon_{\alpha}, 0}, \cdots, \Lambda_{\varepsilon_{\alpha}, 0}}_{N}\}$.

It is known that the diagonal elements of $\boldsymbol{\Lambda}_{\alpha}$ are the power weighting coefficients of sub-carrier $k$, which can be depicted as $\Lambda_{\varepsilon_{\alpha}, 0}$ and the other elements are power leakage weighting coefficients caused by the MCFO, which is expressed as $\Pi_{\alpha}$ with elements of:

$$
\Pi_{\alpha}[k, l]=\left\{\begin{array}{cc}
\Lambda_{\alpha}[k, l] & k \neq l \\
0 & k=l
\end{array}\right.
$$

From formula (3), it can be observed that due to the existence of MCFO, not only ICI, but also MAI emerges, and the orthogonality of SFBC is destroyed. Consequently, the SFBC decode is much more complicated than conventional methods, which should be processed with an efficient MCFO compensation filter.

\subsection{Proposed MCFO compensation algorithm}

It is assumed that the multiple carrier frequency offsets and channel transfer functions of two links are ideally estimated by using the properties transmitted known pilots (see [21] and references therein). In the scenario in which HAPS stations 


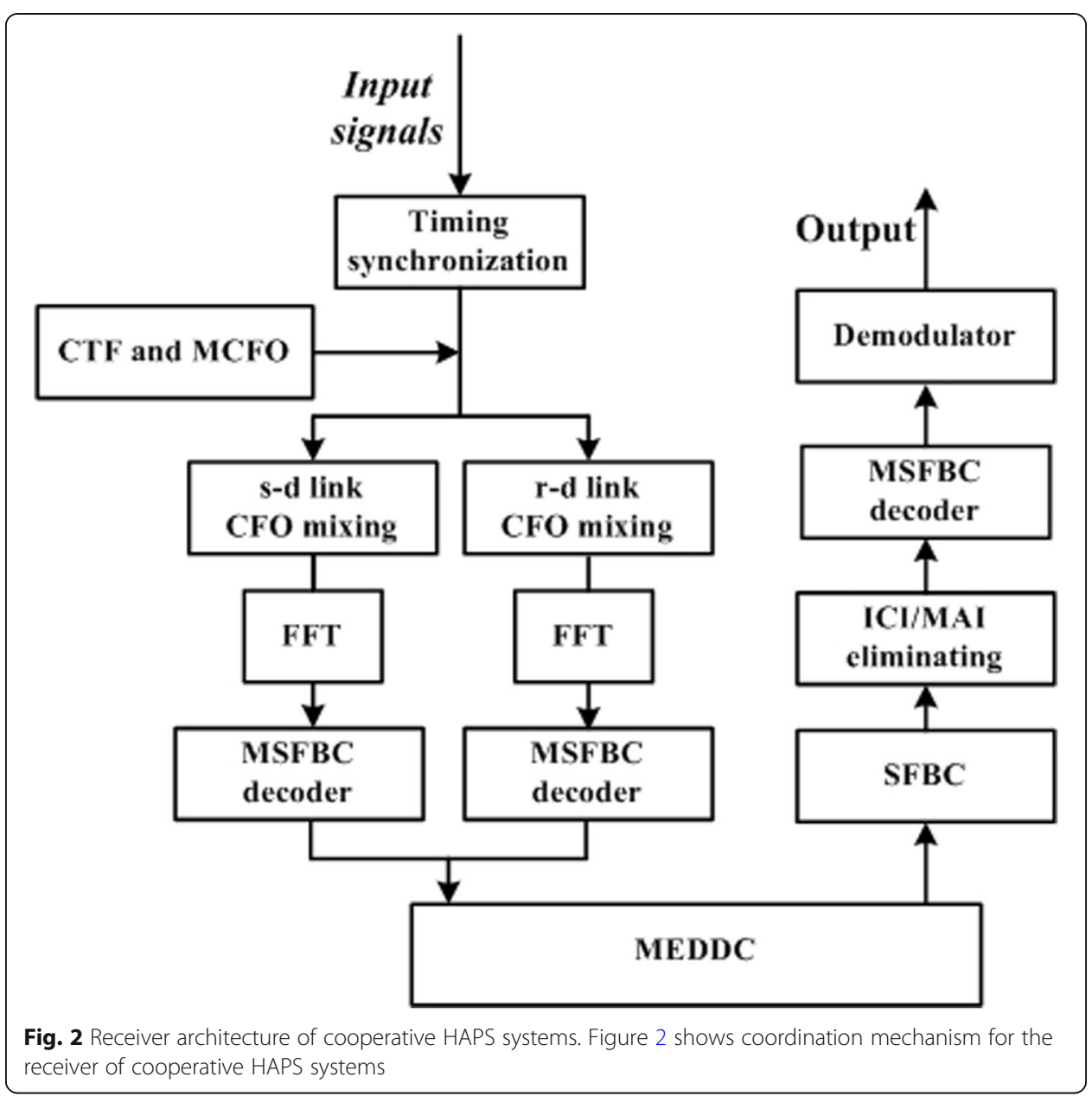

collaborate, the measurement of simple mixing procedure has no effect on multiple frequency offsets compensation, as performed in a single out system [22] (Fig. 2).

\subsubsection{MCFO mixing and modified SFBC decoding}

As shown in formula (3), the received signal is interfered by the interference signal from both source-device link and assist-device link. In order guarantee the performance of SFBC, the received signals should be mixed with the two link's CFO independently as:

$$
\begin{aligned}
& \mathbf{y}_{s d}=\boldsymbol{\Xi}_{s d}^{-1} \cdot \mathbf{y}_{d}=\mathbf{x}_{r d} \cdot \mathbf{h}_{s d}+\boldsymbol{\Xi}_{s d}^{-1} \cdot \boldsymbol{\Xi}_{r d} \cdot \mathbf{x}_{r d} \cdot \mathbf{h}_{r d}+\mathbf{w}_{s d}^{\prime} \\
& \mathbf{y}_{r d}=\boldsymbol{\Xi}_{r d}^{-1} \cdot \mathbf{y}_{d}=\boldsymbol{\Xi}_{r d}^{-1} \cdot \boldsymbol{\Xi}_{s d} \cdot \mathbf{x}_{r d} \cdot \mathbf{h}_{s d}+\mathbf{x}_{r d} \cdot \mathbf{h}_{r d}+\mathbf{w}_{r d}
\end{aligned}
$$

where $\alpha=s d, r d$. If we transform the above formula to frequency domain, the expression can be:

$$
\mathbf{Y}_{\alpha}=\mathbf{F}_{N} \mathbf{y}_{\alpha}
$$

in which the signals on the successive two sub-carriers $k$ and $k+1$ are repressed as: 


$$
\begin{aligned}
& Y_{\alpha, k} \\
& =\left(X_{k} H_{s d, k} \Lambda_{\varepsilon_{s d}-\varepsilon_{\alpha}, 0}+X_{k+1} H_{r d, k} \Lambda_{\varepsilon_{r d}-\varepsilon_{\alpha}, 0}\right) \\
& +\left(\sum_{\substack{l=0 \\
l \neq k}}^{N-1} X_{l} H_{s d, l} \Lambda_{\varepsilon_{s d}-\varepsilon_{\alpha}, l-k}+\sum_{\substack{l=0 \\
l \neq k}}^{N-1} X_{l+1} H_{r d, l} \Lambda_{\varepsilon_{r d}-\varepsilon_{\alpha}, l-k}\right)+W_{s d, k} \\
& Y_{\alpha, k+1} \\
& =\left(-X_{k+1}^{*} H_{s d, k} \Lambda_{\varepsilon_{s d}-\varepsilon_{\alpha}, 0}+X_{k}^{*} H_{r d, k} \Lambda_{\varepsilon_{r d}-\varepsilon_{\alpha}, 0}\right) \\
& +\left(\sum_{\substack{l=0 \\
l \neq k+1}}^{N-1}-X_{l+1}^{*} H_{s d, l} \Lambda_{\varepsilon_{s d}-\varepsilon_{\alpha}, l-k}+\sum_{\substack{l=0 \\
l \neq k+1}}^{N-1} X_{l}^{*} H_{r d, l} \Lambda_{\varepsilon_{r d}-\varepsilon_{\alpha}, l-k}\right)+W_{r d, k+1}
\end{aligned}
$$

Because of the existing of ICI coefficients, the conventional SFBC decoder is ill-fitted and designed. In the algorithm, the decoded signals on successive two sub-carriers are defined as follows:

$$
\begin{aligned}
& \hat{X}_{\alpha, k}=\frac{Y_{\alpha, k} \hat{H}_{s d, k}^{*} \Lambda_{\varepsilon_{s d}-\varepsilon_{\alpha}, 0}^{*}+Y_{\alpha, k+1}^{*} \hat{H}_{r d, k} \Lambda_{\varepsilon_{r d}-\varepsilon_{\alpha}, 0}}{\left(\left|\hat{H}_{s d, k}\right|^{2} \cdot\left|\Lambda_{\varepsilon_{s d}-\varepsilon_{\alpha}, 0}\right|^{2}+\left|\hat{H}_{r d, k}\right|^{2} \cdot\left|\Lambda_{\varepsilon_{r d}-\varepsilon_{\alpha}, 0}\right|^{2}\right)} \\
& \hat{X}_{\alpha, k+1}=\frac{Y_{\alpha, k} \hat{H}_{r d, k}^{*} \Lambda_{\varepsilon_{r d}-\varepsilon_{\alpha}, 0}^{*}-Y_{\alpha, k+1}^{*} \hat{H}_{s d, k} \Lambda_{\varepsilon_{s d}-\varepsilon_{\alpha}, 0}}{\left(\left|\hat{H}_{s d, k}\right|^{2} \cdot\left|\Lambda_{\varepsilon_{s d}-\varepsilon_{\alpha}, 0}\right|^{2}+\left|\hat{H}_{r d, k}\right|^{2} \cdot\left|\Lambda_{\varepsilon_{r d}-\varepsilon_{\alpha}, 0}\right|^{2}\right)}
\end{aligned}
$$

\subsubsection{Minimum Euclidean Distance Decision Criterion}

As is shown in formula (9), two signals, i.e., $\hat{\mathbf{X}}_{s d}$ and $\hat{\mathbf{X}}_{r d}$, are obtained. But they are both not accurate enough. As a result, there should be a suitable criterion to decide the reliability. In this paper, the criterion is chosen as Minimum Euclidean Distance Decision Criterion (MEDDC). With this criterion, we can compare the Euclidean distances from the two signals to their respectively nearest constellation point to select the output, which can be written as:

$$
\hat{X}_{M E D D C, k}=\underset{\vartheta_{i}}{\arg \min }\left(\left\|\hat{X}_{\alpha, k}-\vartheta_{i}\right\|\right)
$$

in which, $\vartheta_{i}$ is the coordinate of the $i$-th constellation point for M-ary modulation, for instance, as shown in Fig. 4. Because the Euclidean distances from $\hat{X}_{s d, k}$ to $\vartheta_{0}$ is less than that from $\hat{X}_{r d, k}$ to $\vartheta_{3}$. Therefore, $\vartheta_{0}$ is selected to be the demodulated result (Fig. 3).

\subsubsection{Interference reconstructing eliminator}

After MEDDC, the ICI and MAI are proposed to be reconstructed and then eliminated. The quasi-transmitted SFBC signals $\hat{\mathbf{X}}_{Q}=\left[\mathbf{X}_{Q}^{s d}, \mathbf{X}_{Q}^{r d}\right]^{T}$ can be re-constructed with $\hat{\mathbf{X}}_{M E D D C}$ through utilizing formula (1). Then, the ICI and MAI components in (8) can be calculated with $\hat{\mathbf{X}}_{Q}$, the estimated ICI and CTF matrixes as: 


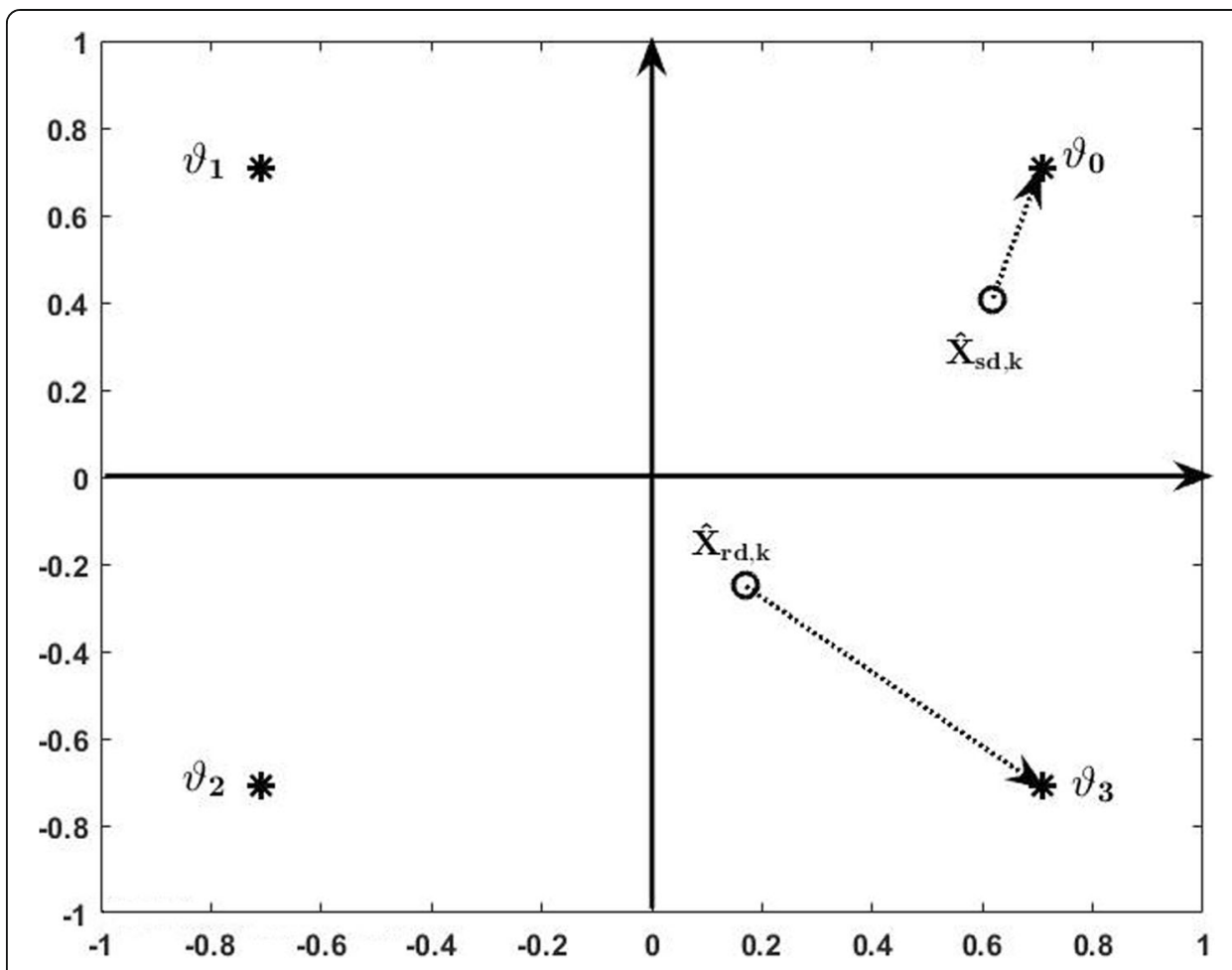

Fig. 3 Euclidean distance comparison. Figure 3 illustrates the Euclidean distance of $\hat{X}_{s d, k}$ and $\hat{X}_{r d, k}$ to its corresponding nearest constellation point

$$
\begin{aligned}
& \mathbf{Y}_{-I C I, \alpha} \\
& =\mathbf{Y}_{\alpha}-\left[\begin{array}{c}
\boldsymbol{\Pi}_{\varepsilon_{s d}-\varepsilon_{\alpha}} \operatorname{diag}\left\{\mathbf{H}_{s d}\right\} \\
\boldsymbol{\Pi}_{\varepsilon_{r d}-\varepsilon_{\alpha}} \operatorname{diag}\left\{\mathbf{H}_{s d}\right\}
\end{array}\right]^{T} \cdot\left[\begin{array}{c}
\mathbf{X}_{Q}^{s d} \\
\mathbf{X}_{Q}^{r d}
\end{array}\right] \\
& \approx \boldsymbol{\Lambda}_{\varepsilon_{s d}-\varepsilon_{\alpha}, 0} \operatorname{diag}\left\{\mathbf{H}_{s d}\right\} \mathbf{X}_{s d}+\boldsymbol{\Lambda}_{\varepsilon_{r d}-\varepsilon_{\alpha}, 0} \operatorname{diag}\left\{\mathbf{H}_{r d}\right\} \mathbf{X}_{r d}+\mathbf{W}_{\alpha}
\end{aligned}
$$

Finally, the transmitted signal can be decoded again through re-employing formula (9) as:

$$
\begin{aligned}
& \hat{X}_{k}=\frac{Y_{-I C I, k} \hat{H}_{s d, k}^{*} \Lambda_{\varepsilon_{s d}-\varepsilon_{\alpha}}^{*}+Y_{-I C I, k+1}^{*} \hat{H}_{r d, k} \Lambda_{\varepsilon_{r d}-\varepsilon_{\alpha}, 0}}{\left(\left|\hat{H}_{s d, k}\right|^{2} \cdot\left|\Lambda_{\varepsilon_{s d}-\varepsilon_{\alpha}, 0}\right|^{2}+\left|\hat{H}_{r d, k}\right|^{2} \cdot\left|\Lambda_{\varepsilon_{r d}-\varepsilon_{\alpha}, 0}\right|^{2}\right)} \\
& \hat{X}_{k+1}=\frac{Y_{-I C I, k} \hat{H}_{r d, k}^{*} \Lambda_{\varepsilon_{r d}-\varepsilon_{\alpha}, 0}^{*}-Y_{-I C I, k+1}^{*} \hat{H}_{s d, k} \Lambda_{\varepsilon_{s d}-\varepsilon_{\alpha}, 0}}{\left(\left|\hat{H}_{s d, k}\right|^{2} \cdot\left|\Lambda_{\varepsilon_{s d}-\varepsilon_{\alpha}, 0}\right|^{2}+\left|\hat{H}_{r d, k}\right|^{2} \cdot\left|\Lambda_{\varepsilon_{r d}-\varepsilon_{\alpha}, 0}\right|^{2}\right)}
\end{aligned}
$$

\subsection{Proposed MCFO compensation algorithm with iterative MEDDC}

The proposed algorithm in this paper can be iteratively utilized to further increase the BER performance. Figure 4 gives the illustration of proposed algorithm with two iterations, in which the ICI eliminating and modified SFBC decoder would be independently implemented after quasi-transmitted SFBC signals reconstructing. At last, the MEDDC is proposed to be utilized again for decreasing the BER of the systems. 


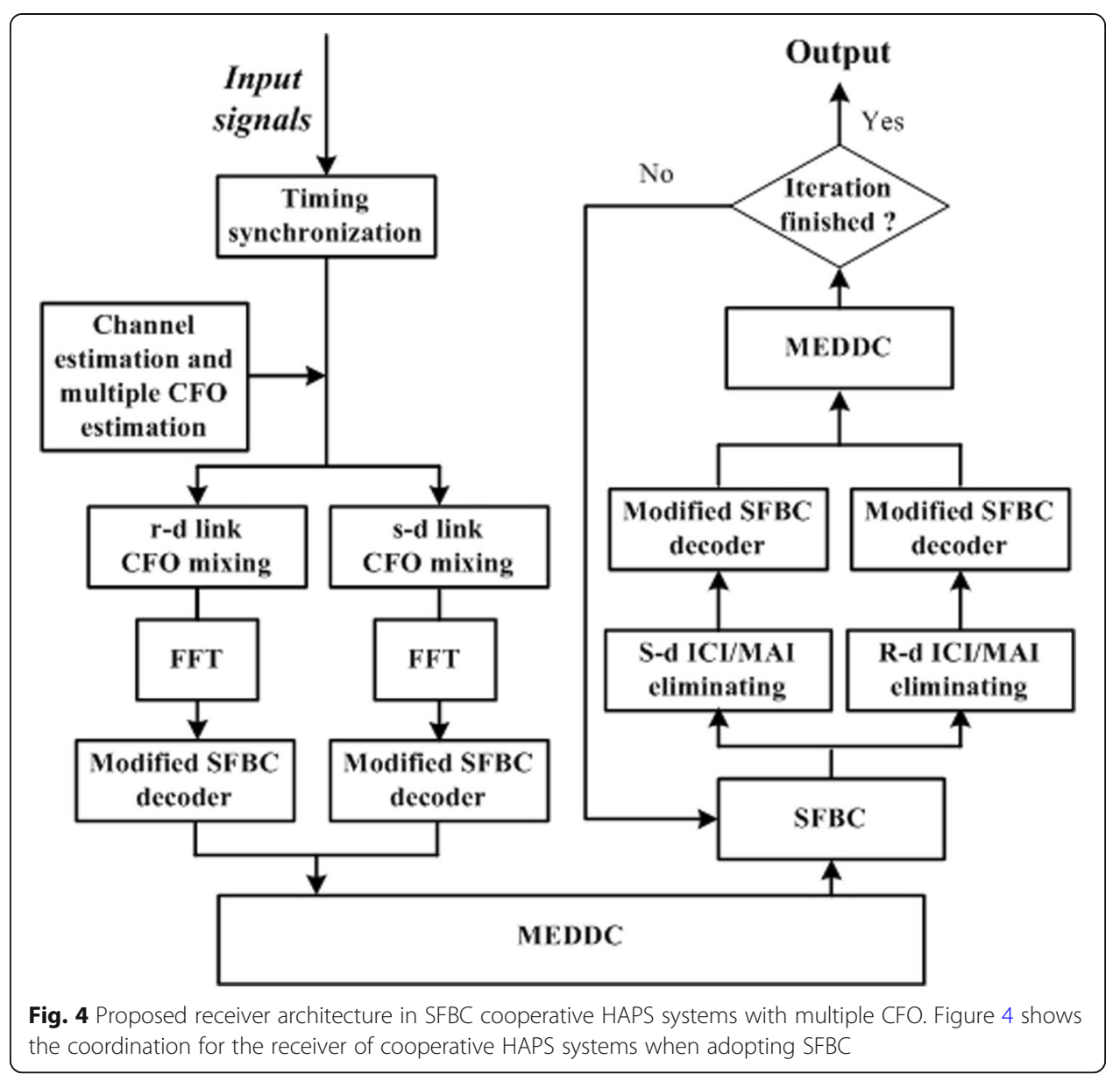

\section{Results and discussion}

\subsection{BER analysis results}

\subsubsection{BER derivation of cooperative communication systems}

It is assumed that each sub-carrier is modulated by M-ary phase shift keying (PSK) with Gray bit mapping. The received signals, which are interfered by MCFO, can be rewritten the Eq. (3) in element expression as:

$$
\begin{aligned}
& Y_{d, k} \\
& =\left(X_{s d, k} H_{s d, k} \Lambda_{\varepsilon_{s d}, 0}+X_{r d, k} H_{r d, k} \Lambda_{\varepsilon_{r d}, 0}\right) \\
& +\left(\sum_{\substack{l=0 \\
l \neq k}}^{N-1} X_{s d, l} H_{s d, l} \Lambda_{\varepsilon_{s d}, l-k}+\sum_{\substack{l=0 \\
l \neq k}}^{N-1} X_{r d, l} H_{r d, l} \Lambda_{\varepsilon_{r d}, l-k}\right)+W_{d, k}
\end{aligned}
$$

Take $\hat{X}_{s d, k}$, for example, its decoded result can be depicted as: 


$$
\begin{aligned}
& \hat{X}_{\alpha, k} \\
& =\frac{Y_{d, k} \hat{H}_{s d, k}^{*} \Lambda_{\varepsilon_{s d}, 0}^{*}+Y_{d, k+1}^{*} \hat{H}_{r d, k} \Lambda_{\varepsilon_{r d}, 0}}{\left|\hat{H}_{s d, k}\right|^{2} \cdot\left|\Lambda_{\varepsilon_{s d}, 0}\right|^{2}+\left|\hat{H}_{r d, k}\right|^{2} \cdot\left|\Lambda_{\varepsilon_{r d}, 0}\right|^{2}} \\
& =X_{s d, k}+\frac{W_{d, k+1}^{*} \hat{H}_{r d, k} \Lambda_{\varepsilon_{r d}, 0}+W_{d, k} \hat{H}_{s d, k}^{*} \Lambda_{\varepsilon_{s d}, 0}^{*}}{\left|\hat{H}_{s d, k}\right|^{2} \cdot\left|\Lambda_{\varepsilon_{s d}, 0}\right|^{2}+\left|\hat{H}_{r d, k}\right|^{2} \cdot\left|\Lambda_{\varepsilon_{r d}, 0}\right|^{2}} \\
& +\frac{\left(\sum_{\substack{l=0 \\
l \neq k}}^{N-1} X_{s d, l}\left(\begin{array}{c}
H_{s d, l} \Lambda_{\varepsilon_{s d}} \hat{H}_{l-k, k}^{*} \Lambda_{\varepsilon_{s d}, 0}^{*}+H_{s d, l}^{*} \Lambda_{\varepsilon_{s d}^{*}}^{*} \hat{H}_{l+1-k} \hat{H}_{r d, k} \Lambda_{\varepsilon_{r d}, 0}
\end{array}\right)\right)}{\left|\hat{H}_{s d, k}\right|^{2} \cdot\left|\Lambda_{\varepsilon_{s d}, 0}\right|^{2}+\left|\hat{H}_{r d, k}\right|^{2} \cdot\left|\Lambda_{\varepsilon_{r d}, 0}\right|^{2}} \\
& +\frac{\left(\sum_{l=0}^{N-1} X_{r d, l}\left(\begin{array}{c}
H_{r d, l} \Lambda_{\varepsilon_{r d}} \hat{H}_{s d, k}^{*} \Lambda_{\varepsilon_{s d}, 0}^{*}-H_{r d, l}^{*} \Lambda_{\varepsilon_{r d}}^{*} \\
l-k
\end{array} \hat{H}_{r d, k} \Lambda_{\varepsilon_{r d}, 0}\right)\right)}{\left|\hat{H}_{s d, k}\right|^{2} \cdot\left|\Lambda_{\varepsilon_{s d}, 0}\right|^{2}+\left|\hat{H}_{r d, k}\right|^{2} \cdot\left|\Lambda_{\varepsilon_{r d}, 0}\right|^{2}}
\end{aligned}
$$

Then, the instantaneous signal to interference plus noise ratio (SINR) of the $k$-th sub-carrier can be written as:

$$
\operatorname{SINR}_{k}=\frac{\sigma_{x}^{2}\left(\left|H_{s d, k} \Lambda_{\varepsilon_{s d}, 0}\right|^{2}+\left|H_{r d, k} \Lambda_{\varepsilon_{r d}, 0}\right|^{2}\right)}{\xi(k)+\sigma_{W}^{2}}
$$

where $\xi(k)$ denotes the ICI/MAI power components. Because the CTF on every subcarriers are assumed to be independent, it can be concluded that $\xi(k)$ is independent with $\left|H_{s d, k} \Lambda_{\varepsilon_{s d}, 0}\right|^{2}+\left|H_{r d, k} \Lambda_{\varepsilon_{r d}, 0}\right|^{2}$. Furthermore, it is easy to know that $\xi(k)$ is nonnegative. Average power allocation is assumed so that $\sigma_{x}^{2}=\sigma_{s}^{2} / M$ with $M$ transmitter nodes. Therefore, according to [20], the instantaneous BER of the $k$ - th sub-carrier can be approximately written as

$$
\begin{aligned}
& P_{c o n}(k) \\
& \simeq \frac{2}{\log _{2} M} Q\left(\sin \frac{\pi}{M} \sqrt{2 \cdot \operatorname{SINR}_{k}}\right) \\
& =\frac{2}{\log _{2} M} Q\left(\sin \frac{\pi}{M} \sqrt{\frac{2 \sigma_{x}^{2}\left(\left|H_{s d, k} \Lambda_{\varepsilon_{s d}, 0}\right|^{2}+\left|H_{r d, k} \Lambda_{\varepsilon_{r d}, 0}\right|^{2}\right)}{\xi(k)+\sigma_{W}^{2}}}\right)
\end{aligned}
$$

In consequence, the average BER for the $k$-th sub-carrier can be expressed:

$$
P_{b}(\xi(k), \gamma)=\int_{0}^{\infty} p\left(z_{k}\right) P_{c o n}(k) d z_{k}
$$

where $z_{k}=\left|H_{s d, k} \Lambda_{\varepsilon_{s d}, 0}\right|^{2}+\left|H_{r d, k} \Lambda_{\varepsilon_{r d}, 0}\right|^{2}, p\left(z_{k}\right)$ denotes the probability density function (PDF) of $z_{k} \cdot P_{b}(\xi(k), \gamma)$ can be obtained as formula (17)

$$
P_{b, p r o p o s e d}(\xi(k), \gamma)
$$

where $\varsigma=\sin ^{2}(\pi / M), \gamma=\sigma_{x}^{2} / \sigma_{W}^{2}, \beta=\sigma_{x}^{2} /\left(\sigma_{W}^{2}+\xi(k)\right)$, and $\vartheta=1 / 4 \beta \varsigma \sigma_{1}^{2}$. The proof is as Appendix 1. 
Finally, we average $P_{b}(\xi(k), \gamma)$ over all sub-carriers with index $k$ as follows to get the BER:

$$
P_{b \_a l l}(\gamma)=\frac{1}{N} \sum_{k=0}^{N-1} P_{b}(\xi(k), \gamma)
$$

\subsubsection{BER analysis}

Theorem 1: The function $P_{b}(\xi(k), \gamma)$ in formula (17) is a monotonically increasing function with respect to $\xi(k)$.

Proof See Appendix 2.

It can be seen from Theorem 1 and in equation $\xi(k) \geq 0$ that when $\xi(k)=0$, the system can achieve the optimal BER performance with specified signal to noise ratio (SNR) $\gamma$. Therefore, the lower bound to the BER performance of the SFBC collaborative communication systems with errors caused by Gaussian noise and multiple CFO can be depicted as formula (19):

$$
P_{b \_a l l}^{L B}=\frac{1}{N} \sum_{k=0}^{N-1} P_{b}(0, \gamma)
$$

where $\varsigma=\sin ^{2}(\pi / M), \gamma=\sigma_{x}^{2} / \sigma_{W}^{2}, \sigma_{1}^{2}=\left|\Lambda_{\varepsilon_{s d}, 0}\right|^{2} \sigma_{H}^{2}$, and $\sigma_{2}^{2}=\left|\Lambda_{\varepsilon_{r d}, 0}\right|^{2} \sigma_{H}^{2}$.

Theorem 2: The function $P_{b}(\xi(k), \gamma)$ in formula (17) is a monotonically decreasing function with respect to $\gamma$.

Proof See Appendix 3.

Through employing Theorem 2, it can be concluded that the BER performance of cooperative systems goes better along with the increase of SNR.

\subsubsection{BER analysis of proposed MCFO compensation algorithm}

When the proposed MCFO compensation algorithm is employed, the received signals are mixed with the one link's CFO before decoding. Therefore, take being mixed with the source-destination link for instance, the MCFO of the two links would be adjusted to be 0 and $\varepsilon_{r d}-\varepsilon_{s d}$. Thus, $\sigma_{1}^{2}$ and $\sigma_{2}^{2}$ in formula (17) would be changed to $\sigma_{H}^{2}$ and $\sigma_{p}^{2}$ $=\left|\Lambda_{\varepsilon_{r d}-\varepsilon_{s d}, 0}\right|^{2} \sigma_{H}^{2}$. Consequently, formula (17) can be rewritten as formula (20):

$$
P_{b}(\xi(k), \gamma)
$$

where $\varsigma=\sin ^{2}(\pi / M), \gamma=\sigma_{x}^{2} / \sigma_{W}^{2}$. As shown in the above formula, the BER performance of the algorithm is not depending on the value of $\varepsilon_{s d}$ and $\varepsilon_{r d}$ but the difference of them. Therefore, it can be concluded that the proposed equalizer not only can compensate the fractional parts of the MCFO but also can compensate the integral parts.

$$
P_{b}(\xi(k), \gamma)=\left\{\begin{array}{cl}
\frac{1}{\log _{2} M} \cdot\left(1-\frac{\left(4 \varsigma \sigma_{1}^{2}+3\left(1 / \gamma+\xi(k) / \sigma_{x}^{2}\right)\right)\left(4 \varsigma \sigma_{1}^{2}\right)^{1 / 2}}{\left(4 \varsigma \sigma_{1}^{2}+2\left(1 / \gamma+\xi(k) / \sigma_{x}^{2}\right)\right)^{3 / 2}}\right) & \left|\Lambda_{\varepsilon_{s d}, 0}\right|=\left|\Lambda_{\varepsilon_{r d}, 0}\right| \\
\frac{1}{\log _{2} M}\left[1+\frac{\sqrt{2 \varsigma}}{\left(\sigma_{2}^{2}-\sigma_{1}^{2}\right)}\left[\sum_{i=1}^{2}(-1)^{i-1} \sigma_{i}^{2} \sqrt{\left.\sigma_{i}^{2} /\left(2 \varsigma \sigma_{i}^{2}+\frac{\xi(k)}{\sigma_{x}^{2}}+\frac{1}{\gamma}\right)\right]}\right]\right. & \left|\Lambda_{\varepsilon_{s d}, 0}\right| \neq\left|\Lambda_{\varepsilon_{r d}, 0}\right|
\end{array}\right.
$$




$$
\begin{aligned}
& P_{b \_ \text {all }}^{L B}=\frac{1}{N} \sum_{k=0}^{N-1} P_{b}(0, \gamma)=\left\{\begin{array}{cc}
\frac{1}{\log _{2} M} \cdot\left(1-\frac{\left(4 \varsigma \sigma_{1}^{2}+3 / \gamma\right)\left(4 \varsigma \sigma_{1}^{2}\right)^{1 / 2}}{\left(4 \varsigma \sigma_{1}^{2}+2 / \gamma\right)^{3 / 2}}\right) & \left|\Lambda_{\varepsilon_{s d}, 0}\right|=\left|\Lambda_{\varepsilon_{r d}, 0}\right| \\
\frac{1}{\log _{2} M}\left[1+\frac{\sqrt{2 \varsigma}}{\left(\sigma_{2}^{2}-\sigma_{1}^{2}\right)}\left[\sum_{i=1}^{2}(-1)^{i-1} \sigma_{i}^{2} \sqrt{\sigma_{i}^{2} /\left(2 \varsigma \sigma_{i}^{2}+\frac{1}{\gamma}\right)}\right]\right] & \mid \Lambda_{\varepsilon_{s d}, 0|\neq| \Lambda_{\varepsilon_{r d}, 0} \mid}
\end{array}\right.
\end{aligned}
$$

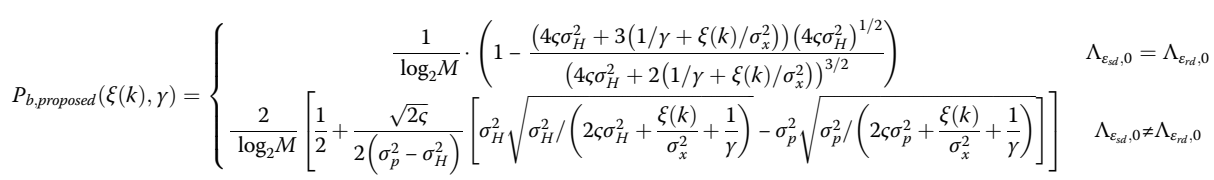

\subsection{Simulation results}

For simulating the wireless channel and mobile environment, the channel B of M.1225 Pedestrian (PB), in which the terminal velocity is $30 \mathrm{~km} / \mathrm{h}$, is utilized. Both fixed and random MCFO are taken into consideration in the simulation. The utilization of fixed MCFO is aimed at verifying the correctness of theoretical analysis, and the random MCFO is for more realistic scenario.

The comparison between the theoretical worst BER performance and that from simulation is depicted in Fig. 5, which shows that the BER curve obtained from the theoretical analysis can gracefully match those in the simulation. We can also see that the BER

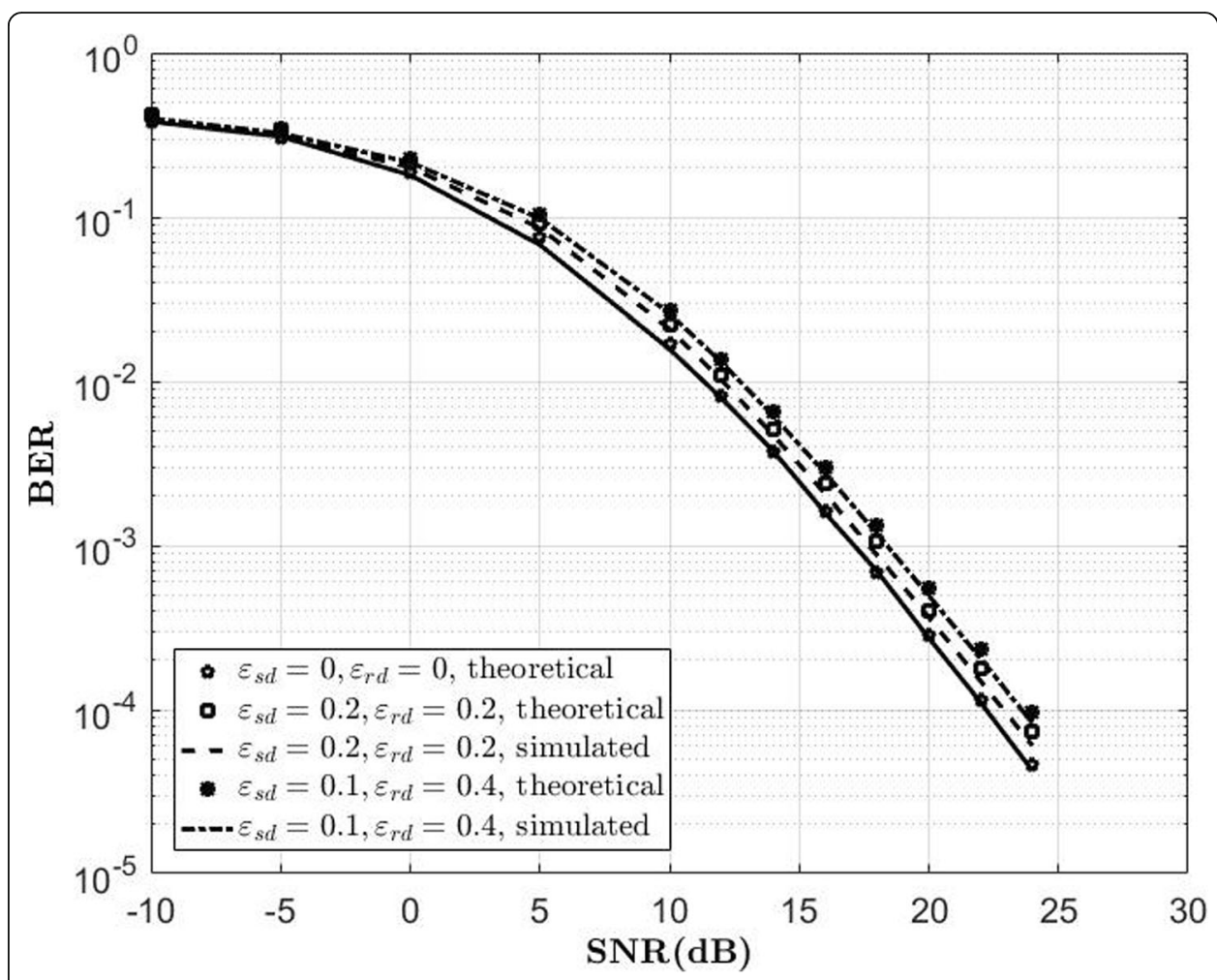

Fig. 5 Comparison of theoretical BER lower bound and the BER performance obtained from the simulation. Figure 5 shows the comparison between the theoretical worst BER performance and that from simulation 
lower bound degrades along with the increasing of $\varepsilon_{\alpha},(\alpha=s d, r d)$. The degradation is with the reason that the SINR shown in (14) is reduced due to power leakage caused by MCFO.

Figure 6 shows the BER of systems without SFBC versus SNR for the proposed MCFO compensation algorithm, where the MCFO are assumed to be random. The results of proposed algorithm with 1 MEDDC and proposed algorithm with 2 MEDDC are presented. The result shows that the proposed algorithm can eliminate the MCFO effectively even when the MCFO is distributed in a relatively large range. It can also be found that when the MCFO range increases, the performance of proposed algorithm with 2 MEDDC is better than that with 1 MEDDC. Therefore, if the MCFO ranges continuously increase, the algorithm is proposed to be employed with more MEDDC iterations (Fig. 7).

When the SNR is fixed at $10 \mathrm{~dB}, 15 \mathrm{~dB}$, and $20 \mathrm{~dB}$ respectively, the relationship between the normalized MCFO difference range and the BER obtained by using the proposed MCFO equalizer is depicted in Fig. 8. It can be observed that the proposed algorithm can reduce the error floor. From the illustration, it is concluded that through using the proposed equalizer, the ICI and ISI introduced by MCFO can be almost eliminated.

In this paper, we propose a new frequency offset compensation algorithm for HAPS IoT systems employing space-frequency block code orthogonal frequency division multiplexing (SFBC-OFDM), which can provide nearly the ideal performance under the condition that the multiple carrier frequency offsets and channel transfer functions of

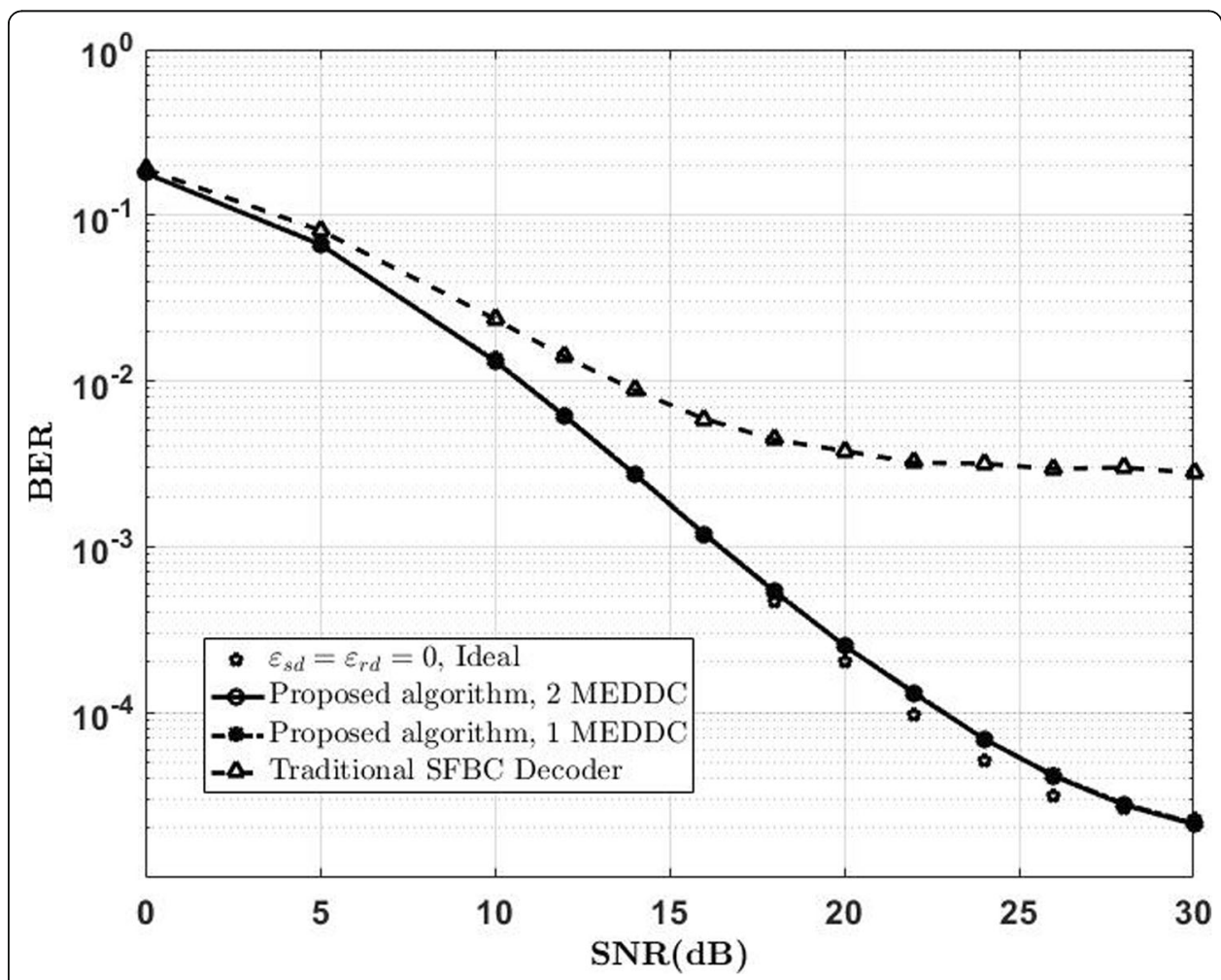

Fig. 6 BER performance of systems without code versus SNR with $\left|\varepsilon_{r d}-\varepsilon_{s d}\right|_{\max }=0.2$. Figure 6 shows the BER performance of systems without code versus SNR for the proposed MCFO compensation algorithm, and the simulation setup is no frequency offset no compensation. $\left|\varepsilon_{r d}-\varepsilon_{s d}\right|_{\text {max }}=0.2$, and PB channel was used 


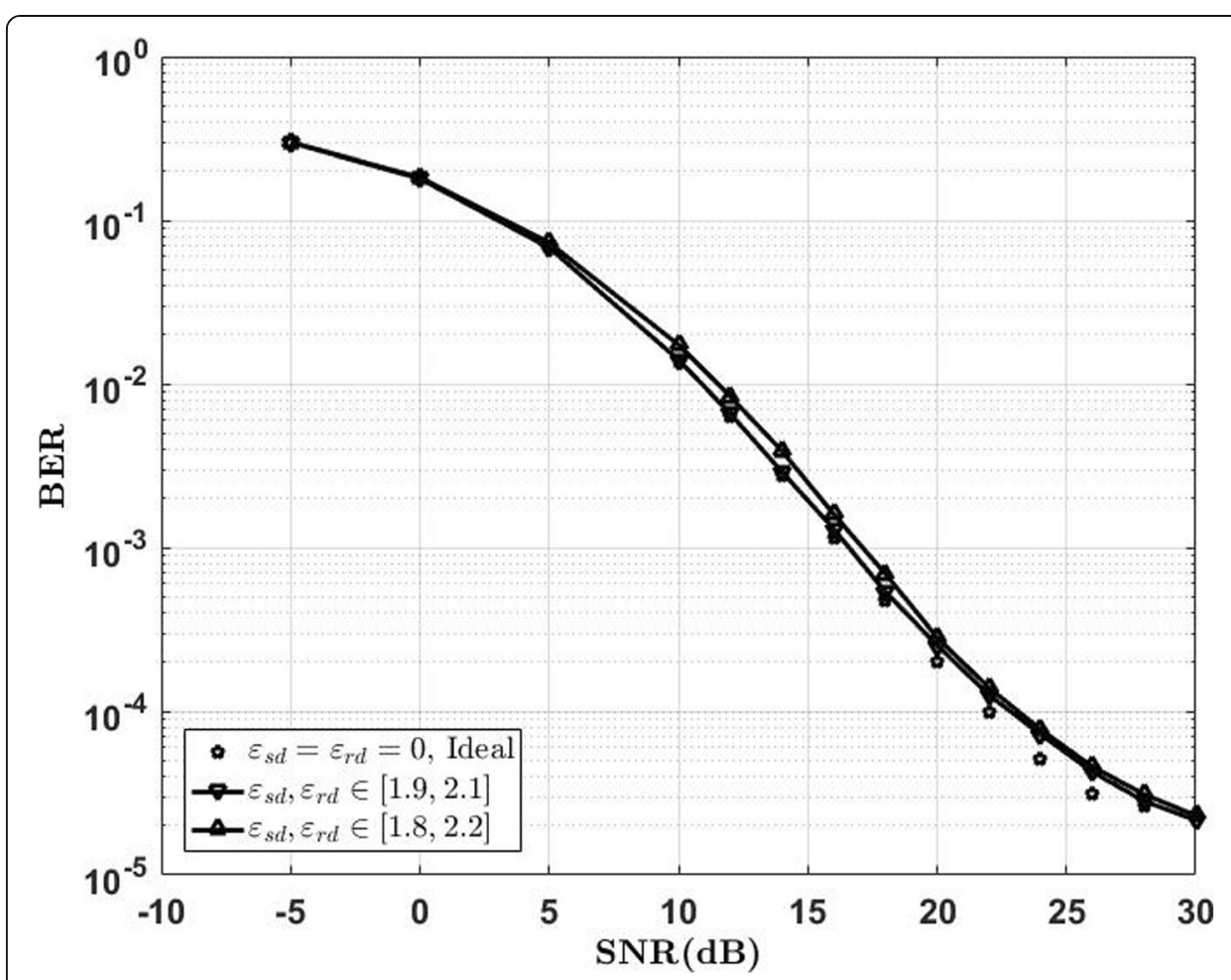

Fig. 7 BER performance of systems without coding versus SNR for the proposed MCFO compensation algorithm. Figure 7 depicts the BER performance of systems without coding versus SNR for the proposed MCFO compensation algorithm, and the simulation setup is no frequency offset no compensation. $\varepsilon_{r d}$ $\varepsilon_{s d} \in[1.9,2.1]$ and $\varepsilon_{r d}, \varepsilon_{s d} \in[1.8,2.2]$, PB channel was used.

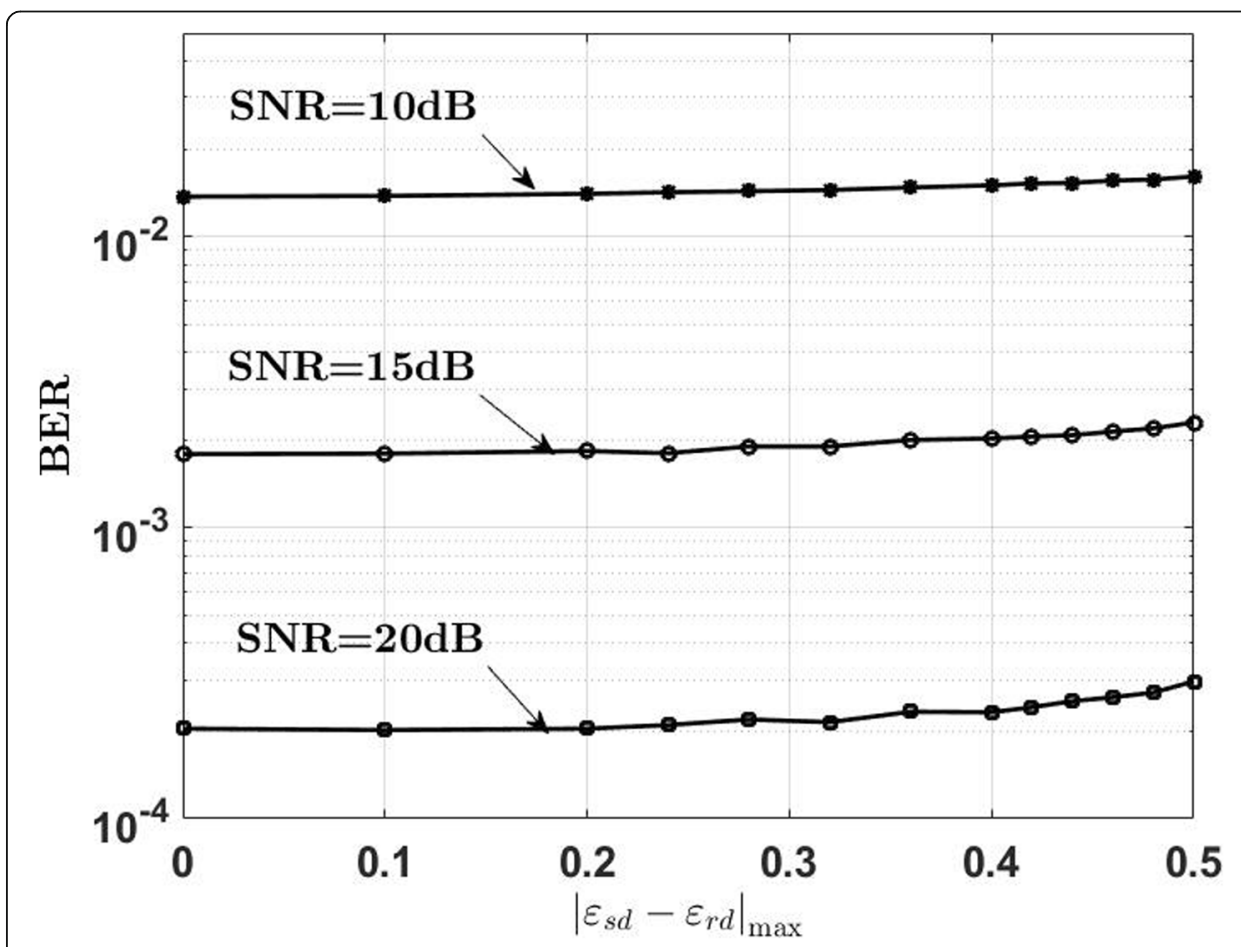

Fig. 8 Effect of $\left|\varepsilon_{s d}-\varepsilon_{r d}\right|_{\text {max }}$ on the BER performance. Figure 8 shows the effect of $\left|\varepsilon_{s d}-\varepsilon_{r d}\right|_{\text {max }}$ on the BER performance of proposed algorithm in collaborative communication systems when PB channel was used 
two links can be ideally estimated by using the properties transmitted known pilots. However, all proposed methods and algorithms are based on the usage of HAPS IoT systems. And much work remains to be done on the usage of HAPS IoT systems.

\section{Conclusion}

In the cooperative HAPS IoT systems, MCFO caused by the multiple carriers may introduce ICI and MAI, which may be impediment in the usage of the systems. To solve the problem caused by ICI and MAI, a MCFO compensation algorithm is proposed in this paper. The proposed algorithm eliminates the interference through utilizing modified SFBC decoder and MEDDC. Furthermore, the BER performance of cooperative communication systems utilizing MCFO is analyzed. Simulation is also carried out to verify the performance of the algorithm. The BER performance loss degraded to around $1 \mathrm{~dB}$ when $\left|\varepsilon_{r d}-\varepsilon_{s d}\right| \leq 0.5$ in multi-path Rayleigh channel. Therefore, it is concluded that the proposed compensation algorithm can effectively solve the problem of frequency offset in cooperative HAPS systems. Thus, the coordination between HAPS stations can provide better communication service in the special scenarios described in the introduction section through coordination by employing the algorithm.

\section{Appendix 1}

Proof of formula (17)

Rewrite the average BER of sub-carrier $k$ as:

$$
P_{b}(\xi(k), \gamma)=\int_{0}^{\infty} p\left(z_{k}\right) P_{c o n}(k) d z_{k}
$$

where $z_{k}=\left|H_{s d, k} \Lambda_{\varepsilon_{s d}, 0}\right|^{2}+\left|H_{r d, k} \Lambda_{\varepsilon_{r d}, 0}\right|^{2}$.

When $\left|\Lambda_{\varepsilon_{s d}, 0}\right|$ is equal to $\left|\Lambda_{\varepsilon_{r d}, 0}\right|, z_{k}$ can be thought as independent chi-square random variable of $4^{\circ}$ with mean value of $2 \sigma_{1}^{2}$, where $\sigma_{1}^{2}=\sigma_{H}^{2}\left|\Lambda_{\varepsilon_{s d}, 0}\right|^{2}=\sigma_{H}^{2}\left|\Lambda_{\varepsilon_{r d}, 0}\right|^{2}$. In consequence, $p\left(z_{k}\right)$ can be expressed as:

$$
p\left(z_{k}\right)=\frac{1}{4 \sigma_{1}^{2}} \cdot z_{k} \cdot e^{-z_{k} / 2 \sigma_{1}^{2}}, \quad z_{k} \geq 0
$$

Therefore, the theoretical expression for BER is as follows:

$$
\begin{aligned}
& P_{b}(\xi(k), \gamma) \\
& =\frac{2}{\log _{2} M} \int_{0}^{\infty} p\left(z_{k}\right) \int_{\sqrt{2 \beta \zeta z_{k}}}^{\infty} \frac{1}{\sqrt{2 \pi}} e^{-y^{2} / 2} d y d z_{k} \\
& =\frac{2}{\log _{2} M} \int_{0}^{\infty} \frac{1}{\sqrt{2 \pi}} e^{-y^{2} / 2} d y\left(\int_{0}^{\frac{y^{2}}{2 \alpha \beta}} p\left(z_{k}\right) d z_{k}\right) \\
& =\frac{2}{\log _{2} M} \int_{0}^{\infty} \frac{1}{\sqrt{2 \pi}} e^{-\frac{y^{2}}{2}}\left(1-\frac{y^{2}}{4 \beta \zeta \sigma_{1}^{2}} \cdot e^{-\frac{y^{2}}{4 \beta \sigma_{1}^{2}}}-e^{-\frac{y^{2}}{4 \beta \sigma_{1}^{2}}}\right) d y \\
& =\frac{2}{\log _{2} M} \cdot \int_{0}^{\infty} \frac{1}{\sqrt{2 \pi}} e^{-\frac{y^{2}}{2}}\left(1-9 y^{2} \cdot e^{-9 y^{2}}-e^{-9 y^{2}}\right) d y
\end{aligned}
$$




$$
\begin{aligned}
& =\frac{2}{\log _{2} M} \cdot\left(\frac{1}{2}-\frac{\vartheta}{2} \cdot\left(\frac{1}{1+2 \vartheta}\right)^{3 / 2}-\frac{1}{2 \sqrt{1+2 \vartheta}}\right) \\
& =\frac{1}{\log _{2} M} \cdot\left(1-\frac{1+3 \vartheta}{(1+2 \vartheta)^{3 / 2}}\right) \\
& =\frac{1}{\log _{2} M} \cdot\left(1-\frac{4 \varsigma \sigma_{1}^{2}+3\left(1 / \gamma+\xi(k) / \sigma_{x}^{2}\right)}{\left(4 \varsigma \sigma_{1}^{2}+2\left(1 / \gamma+\xi(k) / \sigma_{x}^{2}\right)\right)^{3 / 2}}\right)
\end{aligned}
$$

where $\varsigma=\sin ^{2}(\pi / M), \gamma=\sigma_{x}^{2} / \sigma_{W}^{2}, \beta=\sigma_{x}^{2} /\left(\sigma_{W}^{2}+\xi(k)\right)$, and $\vartheta=1 / 4 \beta \varsigma \sigma_{1}^{2}$.

When $\left|\Lambda_{\varepsilon_{s d}, 0}\right|$ is equal to $\left|\Lambda_{\varepsilon_{r d}, 0}\right|, z_{k}$ can be rewritten as:

$$
\begin{aligned}
& z_{k} \\
& =\left|H_{s d, k}\right|^{2}+\left|H_{r d, k}\right|^{2} \\
& =\underbrace{\left|\Lambda_{\varepsilon_{s d}, 0}\right|^{2} \mathfrak{R}^{2}\left(H_{s d, k}\right)+\left|\Lambda_{\varepsilon_{s d}, 0}\right|^{2} \mathfrak{S}^{2}\left(H_{s d, k}\right)}_{X_{z}} \\
& +\underbrace{\left|\Lambda_{\varepsilon_{r d}, 0}\right|^{2} \mathfrak{R}^{2}\left(H_{r d, k}\right)+\left|\Lambda_{\varepsilon_{r d d}, 0}\right|^{2} \mathfrak{S}^{2}\left(H_{r d, k}\right)}_{Y_{z}}
\end{aligned}
$$

in which $X_{z}$ and $Y_{z}$ can be thought as independent chi-square random variables of $2^{\circ}$ with mean value of $\sigma_{1}^{2}=\left|\Lambda_{\varepsilon_{s d}, 0}\right|^{2} \sigma_{H}^{2}$ and $\sigma_{2}^{2}=\left|\Lambda_{\varepsilon_{r d}, 0}\right|^{2} \sigma_{H}^{2}$. Therefore, $p\left(z_{k}\right)$ can be calculated as:

$$
\begin{aligned}
& p\left(z_{k}\right) \\
& =\int_{-\infty}^{\infty} p_{X_{z}}(x) p_{Y_{z}}\left(z_{k}-x\right) d x \\
& =\int_{0}^{z_{k}} p_{X_{z}}(x) p_{Y_{z}}\left(z_{k}-x\right) d x=\int_{0}^{z_{k}} \frac{1}{2 \sigma_{1}^{2}} e^{-\frac{x}{2 \sigma_{1}^{2}}} \frac{1}{2 \sigma_{2}^{2}} e^{-\frac{z_{k}-x}{2 \sigma_{2}^{2}}} d x \\
& =\frac{1}{2\left(\sigma_{1}^{2}-\sigma_{2}^{2}\right)}\left(e^{-\frac{z_{k}}{2 \sigma_{1}^{2}}}-e^{-\frac{z_{k}}{2 \sigma_{2}^{2}}}\right), \quad z_{k} \geq 0
\end{aligned}
$$

Therefore, the BER can be obtained as:

$$
\begin{aligned}
& P_{b}(\xi(k), \gamma) \\
& =\frac{2}{\log _{2} M} \int_{0}^{\infty} p\left(z_{k}\right) \int_{\sqrt{2 \beta \zeta z_{k}}}^{\infty} \frac{1}{\sqrt{2 \pi}} e^{-y^{2} / 2} d y d z_{k} \\
& =\frac{2}{\log _{2} M} \int_{0}^{\infty} \frac{1}{\sqrt{2 \pi}} e^{-y^{2} / 2} d y\left(\int_{0}^{\frac{y^{2}}{2 \beta \beta}} p\left(z_{k}\right) d z_{k}\right) \\
& =\frac{2}{\log _{2} M} \int_{0}^{\infty} \frac{1}{\sqrt{2 \pi}} e^{-\frac{\gamma^{2}}{2}}\left[1+\frac{\left(\sigma_{2}^{2} e^{-\frac{y^{2}}{4 \alpha \beta \sigma_{2}^{2}}}-\sigma_{1}^{2} e^{-\frac{y^{2}}{4 \beta \sigma_{\sigma_{2}^{2}}}}\right)}{\left(\sigma_{2}^{2}-\sigma_{1}^{2}\right)}\right] d y=\frac{1}{\log _{2} M}\left[1+\frac{\sum_{i=1}^{2}(-1)^{i-1} \sigma_{i}^{2} \sqrt{2 \varsigma \beta \sigma_{i}^{2} /\left(2 \varsigma \beta \sigma_{i}^{2}+1\right)}}{\left(\sigma_{2}^{2}-\sigma_{1}^{2}\right)}\right] \\
& =\frac{1}{\log _{2} M}\left[1+\frac{\sqrt{2 \zeta} \sum_{i=1}^{2}(-1)^{i-1} \sqrt{\sigma_{i}^{6} /\left(2 \zeta \sigma_{i}^{2}+\frac{\xi(k)}{\sigma_{x}^{2}}+\frac{1}{\gamma}\right)}}{\left(\sigma_{2}^{2}-\sigma_{1}^{2}\right)}\right]
\end{aligned}
$$

where $\varsigma=\sin ^{2}(\pi / M), \beta=\sigma_{x}^{2} /\left(\sigma_{W}^{2}+\xi(k)\right)$, and $\gamma=\sigma_{x}^{2} / \sigma_{W}^{2}$.

Therefore, formula (17) can be proved by combining (25) and (27). 


\section{Appendix 2}

Proof of Theorem 1

As shown in formula (17), when $\left|\Lambda_{\varepsilon_{s d}, 0}\right|$ is equal to $\left|\Lambda_{\varepsilon_{r d}, 0}\right|$, we firstly consider the equation

$$
P_{b}(k, \vartheta(\gamma))=\frac{1}{\log _{2} M} \cdot\left(1-\frac{1+3 \vartheta}{(1+2 \vartheta)^{3 / 2}}\right)
$$

where $\vartheta(\xi(k))=\frac{1}{4 \varsigma \sigma_{1}^{2}}\left(\frac{1}{\gamma}+\frac{\xi(k)}{\sigma_{x}^{2}}\right)$; then,

$$
\frac{d\left(P_{b}(\vartheta)\right)}{d \vartheta}=\frac{3 \vartheta}{2(1+2 \vartheta)^{5 / 2}}>0
$$

Furthermore, $\vartheta(\xi(k))=\frac{1}{4 \varsigma \sigma_{1}^{2}}\left(\frac{1}{\gamma}+\frac{\xi(k)}{\sigma_{x}^{2}}\right)$ is also a monotonically increasing function to $\xi(k)$. Therefore, it can be concluded that $P_{b}(k, \vartheta(\gamma))$ increases along with the increase of $\xi(k)$.

When $\left|\Lambda_{\varepsilon_{s d}, 0}\right|$ is unequal to $\left|\Lambda_{\varepsilon_{r d}, 0}\right|$

$$
\begin{aligned}
& d\left\{P_{b}(\xi(k), \gamma)\right\} / d(\xi(k) \\
= & \frac{\sqrt{2 \zeta}}{\log _{2} M\left(\sigma_{2}^{2}-\sigma_{1}^{2}\right)} \cdot \sum_{i=1}^{2}(-1)^{i-1} \frac{d\left\{-\frac{\sigma_{i}^{3}}{2 \sigma_{x}^{2}}\left(2 \varsigma \sigma_{i}^{2}+\frac{\xi(k)}{\sigma_{x}^{2}}+\frac{1}{\gamma}\right)^{-\frac{3}{2}}\right\}}{d \xi(k)}=\frac{\sqrt{2 \varsigma}}{2 \sigma_{x}^{2} \log _{2} M\left(\sigma_{2}^{2}-\sigma_{1}^{2}\right)} \sum_{i=1}^{2}(-1)^{i}\left(2 \varsigma+\frac{\xi(k)}{\sigma_{i}^{2} \sigma_{x}^{2}}+\frac{1}{\sigma_{i}^{2} \gamma}\right)^{-\frac{3}{2}}>0 .
\end{aligned}
$$

Therefore, it can be concluded that the function $P_{b}(\xi(k), \gamma)$ in formula (17) increases along with the increase of $\xi(k)$.

\section{Appendix 3}

Proof of Theorem 2

From formula (17), it is easy to know that the monotonicity of $P_{b}(\xi(k), \gamma)$ with respect to $1 / \gamma$ is the same with respect to $\xi(k)$. Thus, it can be similarly proved that $P_{b}(\xi(k), \gamma)$ decreases along with the increase of $\gamma$.

\section{Abbreviations}

BER: Bit error ratio; CP: Cyclic prefix; CTF: Channel transform function; HAPS: High Altitude Platform Station; ICI: Intercarrier interference; IOT: Internet of Things; ISI: Inter-symbol interference; MAI: Multiple access interference; MCFO: Multiple carrier frequency offsets; MEDDC: Minimum Euclidean Distance Decision Criterion; NB-loT: Narrow Band Internet of Things; OFDM: Orthogonal frequency division multiplexing; PB: Channel B of M.1225 Pedestrian; PDF: Probability density function; PSK: Phase shift keying; SFBC: Space-frequency block code; SFBC-OFDM: Spacefrequency block code orthogonal frequency division multiplexing; SINR: Signal to interference plus noise ratio; SNR: Signal to noise ratio

\section{Acknowledgements}

Not applicable

\section{Authors' contributions}

In this paper, Yanyan Zhang conceived and designed the study. And the paper was written by Yanyan Zhang and Baocong Wang. Xing Li, Na Liu, Wenjuan Ji, and Xin Li reviewed and edited the original manuscript. All authors read and revised the manuscript.

Funding

The work in this paper was supported by China Mobile Group Design Institute Co., Ltd.

\section{Availability of data and materials}

The corresponding author shall keep the analysis and simulation data set. If necessary, the data set can be requested from the corresponding author according to reasonable requirements.

\section{Competing interests}

The authors declare that they have no competing interests. 
Received: 27 January 2020 Accepted: 6 July 2020

Published online: 01 August 2020

\section{References}

1. Sivarajan, Kumar N. "LTE for rural applications and humanitarian relief." International Conference on Wireless Technologies for Humanitarian Relief 2011.

2. R.S. Wolff, E. Andrews, Broadband access, citizen en-franchisement, and telecommunications services in rural and remote areas: a report from the American frontier. IEEE Commun. Mag. 48(5), 128-135 (2010)

3. Takaki, Ricardo, et al. Integration between LTE and satellite networks. Long Term Evolution. Springer International Publishing, 2016.

4. H. Abdzadeh-Ziabari, W.P. Zhu, M.N.S. Swamy, Joint carrier frequency offset and doubly selective channel estimation for MIMO-OFDMA uplink with Kalman and particle filtering. IEEE Transactions on Signal Processing PP. 99, 1-1 (2018)

5. J. Pokorny et al., Concept design and performance evaluation of UAV-based backhaul link with antenna steering. J. Commun. Netw. 20(5), 473-483 (2018)

6. MITRA, and MONOJIT, Satellite Communication. lete Journal of Education 38(1), 3-10 (2018)

7. C. Joo, J. Choi, Low-delay broadband satellite communications with high-altitude unmanned aerial vehicles. J. Commun. Netw. 20(1), 102-108 (2018)

8. "Satellite communications integration with terrestrial networks." China Communications v.15.08(2018):32-48.

9. Ahuna, M. N and T. J. Afullo. "Effects of storm attenuation over satellite links in sub-tropical Africa." 2018 Progress in Electromagnetics Research Symposium (PIERS-Toyama) (2018). doi:10.23919/PIERS.2018.8597964.

10. A.K. Widiawan, R. Tafazolli, High altitude platform station (HAPS): a review of new infrastructure development for future wireless communications. Wirel. Pers. Commun. 42(3), 387-404 (2007)

11. M.D. Zakaria et al., Exploiting user-centric joint transmission - coordinated multipoint with a high altitude platform system architecture. IEEE Access 7, 38957-38972 (2019)

12. Sheikh, Javaid A. , et al. "Impact of antenna and beam selection based sectored relay planning for performance evaluation of 4G LTE-A tri sectored cell." Digital Communications and Networks (2017):S2352864816300864.

13. Hu, Sha, et al. "Improving the performance of OTDOA based positioning in NB-IoT systems." GLOBECOM 2017 - 2017 IEEE Global Communications Conference IEEE, 2018.

14. D. Veronesi and D.L. Goeckel, "Multiple frequency offset compensation in cooperative wireless systems," in Proc. IEEE Global Telecommun. Conf., San Francisco, California, USA, Nov. 2006

15. Rao, R. S. Suriavel, and P. Malathi. "A low complex cuckoo search optimizer based OFDM carrier frequency offset estimation for 5G wireless technology." International Journal of Dynamics and Control (2019). doi: 10.1007/s40435-01900526-9.

16. Van Yem, Vu, and T. T. Huong. "ICl mitigation by estimation of double carrier frequency offsets in high-speed-railway communication systems for smart cities." Mobile Networks and Applications (2018).

17. Z. Qifei et al., Joint channel and carrier frequency offset estimation and correction for coherent optical FBMC/OQAM system. Acta Opt. Sin. 38(2), 0206003 (2018)

18. Gao, Mingjun, et al. "Blind identification of SFBC-OFDM signals using subspace decompositions and random matrix theory." IEEE Transactions on Vehicular Technology (2018):1-1.

19. J. Han, W. Shi, G. Leus, Space-frequency coded orthogonal signal-division multiplexing over underwater acoustic channels. The Journal of the Acoustical Society of America 141(6), EL513-EL518 (2017)

20. Tranter, W, et al. A simple transmit diversity technique for wireless communications. 2007.

21. Veronesi, Daniele, and D. Goeckel. "Multiple frequency offset compensation in cooperative wireless systems." Proceedings of the Global Telecommunications Conference, 2006. GLOBECOM '06, San Francisco, CA, USA, 27 November - 1 December 2006 IEEE, 2006.

22. Y. Dong-Dong et al., Design of the matched chaotic stimulation for fault detection of the single input single output system. Acta Phys. Sin. 63(12), 120508-120130 (2014)

\section{Publisher's Note}

Springer Nature remains neutral with regard to jurisdictional claims in published maps and institutional affiliations.

\section{Submit your manuscript to a SpringerOpen ${ }^{\circ}$ journal and benefit from:}

- Convenient online submission

- Rigorous peer review

- Open access: articles freely available online

- High visibility within the field

- Retaining the copyright to your article

Submit your next manuscript at $\boldsymbol{\Delta}$ springeropen.com 